\title{
SPECTRAL PERTURBATION BOUNDS FOR SELFADJOINT OPERATORS I
}

\author{
KREŠIMIR VESELIĆ
}

\begin{abstract}
We give general spectral and eigenvalue perturbation bounds for a selfadjoint operator perturbed in the sense of the pseudo-Friedrichs extension. We also give several generalisations of the aforementioned extension. The spectral bounds for finite eigenvalues are obtained by using analyticity and monotonicity properties (rather than variational principles) and they are general enough to include eigenvalues in gaps of the essential spectrum.
\end{abstract}

Mathematics subject classification (2000): 47A55.

Key words and phrases: Construction of selfadjoint operators, indefinite forms, perturbation od eigenvalues, relative errors.

\section{REFERENCES}

[1] J. Dolbeault, M. J. EsteBAn, E. SÈRÈ, Variational characterisation for eigenvalues of Dirac operators, Preprint mp-arc 98-177, to appear in Calc. Var. and PDE.

[2] W. G. FARIS, Self-adjoint operators, Lecture Notes in Mathematics, Vol. 433, Springer-Verlag, BerlinNew York, 1975.

[3] T. Kato, Perturbation Theory for Linear Operators, Springer, Berlin 1966.

[4] M. GRIESEMER, R. T. LEWIS, H. SiEDENTOP, A minimax principle for eigenvalues in spectral gaps: Dirac operators with Coulomb potentials, Documenta Mathematica 4 (1999) 275-283.

[5] L. GrubišIĆ, V. Kostrykin, K. A. MaKarov, K. Veselić, On the perturbation theory for quadratic forms, in preparation.

[6] G. NENCIU, Self-adjointness and invariance of the essential spectrum for Dirac operators defined as quadratic forms, Comm. Math. Phys. 48 (1976) 235-247.

[7] B. Thaller, The Dirac Equation, Springer 1992.

[8] K. VeSELIĆ, Perturbation theory for the eigenvalues of factorised symmetric matrices, LAA 309 (2000) 85-102.

[9] K. VESELIĆ, I. SLAPNIČAR, Floating point perturbations of Hermitian matrices, Linear Algebra Appl. 195 (1993) 81-116.

[10] M. WinkLMeIER, The Angular Part of the Dirac Equation in the Kerr-Newman Metric: Estimates for the Eigenvalues, $\mathrm{Ph}$. D. Thesis 2005.

[11] R. WÜst, Dirac operations with strongly singular potentials. Distinguished self-adjoint extensions constructed with a spectral gap theorem and cut-off potentials, Math. Z. 152 (1977), no. 3, 259-271. 